\title{
Smartphone-based application improves the detection of retinoblastoma
}

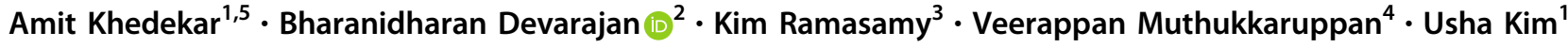

Received: 6 March 2018 / Revised: 29 November 2018 / Accepted: 20 December 2018 / Published online: 11 January 2019

(c) The Royal College of Ophthalmologists 2019

\begin{abstract}
Purpose To improve and validate the smartphone-based leukocoria detection application so that non-ophthalmologists could make use of the smartphone for early detection of Retinoblastoma (RB) in young children without anesthesia and pharmacological dilatation of the pupil.

Methods Two apps, MDEyeCare and CRADLE, developed for red reflex based leukocoria detection were used in iPhone 6s. MDEyeCare methodology was modified with respect to ambient lighting, the distance between camera and eye and different gazes for better performance. We analyzed totally 34 eyes of 23 RB patients and four normal children. Each of the RB patients was confirmed with clinical examination and radiological investigations.

Results Modification in the methodology of MDEyeCare app could detect the leukocoria in early stages of RB (50\% of Group B, $83 \%$ of Group C). In late stages (Group D and E), $100 \%$ of tumors were detected. The CRADLE app failed to provide adequate leukocoria detection except four late stage RB eyes. Among the 14 normal eyes ( 6 from unilateral RB and eight from normal children), pseudo-leukocoria was observed in three eyes only at lateral gaze even with MDEyeCare app. Conclusion Improved methodology in smartphone-based app enhanced the detection of RB and this may translate into better outcome after treatment with respect to vision salvage.
\end{abstract}

\section{Introduction}

Retinoblastoma (RB) is the most common intraocular tumor of childhood under the age of five. It is estimated that 5000 to 8000 children develop RB worldwide every year [1]. The incidence rate of RB in India is 9.6 per million in the age

Supplementary information The online version of this article (https:// doi.org/10.1038/s41433-018-0333-7) contains supplementary material, which is available to authorized users.

Bharanidharan Devarajan

bharanid@gmail.com

bharani@aravind.org

1 Department of Orbit, Oculoplasty and Oncology, Aravind Eye Hospital, Madurai, India

2 Department of Bioinformatics, Aravind Medical Research Foundation, Madurai, India

3 Department of Retina, Aravind Eye Hospital, Madurai, India

4 Advisor-Research, Aravind Medical Research Foundation, Madurai, India

5 Present address: Khedekar's Eye Clinic, Mumbai, India group of $0-4$ [2]. RB is more commonly diagnosed by childhood screening in developed countries and thus has high survival rate $(85-98 \%)$ [3, 4] compared to $50 \%$ in India [5]. Our retrospective study (unpublished) showed $88 \%$ survival rate; however, the vision salvage was only $28 \%$. Shields et al., [6] suggested that it is possible to increase vision salvage to $100 \%$ if $\mathrm{RB}$ is detected and treated at early stages.

White reflex in RB patients is considered as leukocoria, which is the most common presenting sign of $\mathrm{RB}$ accounting for almost $60 \%$. Abramson et al., [7] demonstrated that leukocoria is more often initially detected by family/friends. Originally, it has been assumed that leukocoria is associated with late stage RB [7]. However, a recent study showed that it could be detected in early stage RB [8]. Therefore, it is important to make healthcare professionals involved in child care to detect leukocoria soon after birth, without dilatation and anesthesia, in order to achieve increased vision salvage.

In photography using conventional camera with flash in the dark/dim light source, red reflex can be seen in the normal eye. Leukocoria can be seen in the eye with tumor due to the reflection of the flash light from the abnormal 
retina in the back of the eye. However, in the modern camera including the cell phone, a red eye reduction feature is included so that red reflex is prevented. With the advent of smartphone technologies, applications (apps) such as CRADLE [8, 9] and MDEyeCare (www.mdeyecare.com) are designed to detect red reflex or leukocoria, depending on the case. However, the decision to recommend an app for $\mathrm{RB}$ detection can have consequences if it is inaccurate or ineffective. For example, a mobile app for assessing melanoma risk had very low sensitivity and inaccuracy in early detection of many melanomas, which can delay the diagnosis and harm users [10]. Thus, these apps require scrutiny and validation with clinical confirmation.

In the present study, we propose to improve the performance of MDEyeCare App with modifications in the photography and validate its performance for early detection of Retinoblastoma (RB) in Indian children without anesthesia and pharmacological dilatation of the pupil.

\section{Materials and methods}

A total of 6 unilateral and 17 bilateral RB patients of both sexes who volunteered were selected during August 2014 and Feb 2016 for this study (Table 1). Six of the bilateral RB patients who underwent enucleation in one eye were also included in this study. Patients, mostly referred to the clinic by family members with leukocoria as presenting sign (Table 1), were initially examined using iPhone-based apps. At first, MDEyeCare app with modified methodology was used to detect the leukocoria as described below. Later, all the images were screened using CRADLE app followed by clinical examination. Eight eyes of four normal children were

Table 1 Details of RB patients

Total number $(\%)$

Sex

Male

Female

$14(61)$

Laterality

Unilateral

$6(26)$

Bilateral

17 (74)

Mean age at the time of presentation

$24.7 \pm 5.4$ month

Presenting sign

Leukocoria

$20(87)$

Poor vision

$2(8)$

Conjunctival nevus

$1(4)$

Referred by

Family

Ophthalmologist

Other

$1(4)$ included in this study. Among them, two were relatives' of adult non-RB patients and two were siblings of RB patients. After getting the informed consent from parents, iPhone $6 \mathrm{~s}$ based apps were used for the leukocoria detection.

The diagnosis of RB was made by thorough clinical examination and radiological investigations (CT/MRI and USG B scan) along with Retcam imaging in Aravind Eye Hospital Madurai, India. For grading of the tumor, tumor was grouped into A, B, C, D, and E based on the tumor size, location and vitreous seeds according to the International Classification of Retinoblastoma [11]. Patients received chemotherapy, radiotherapy, and surgical intervention as per the protocols. The present study was approved by the Institutional Ethics Committee of Aravind Eye Hospital, India (Registration Number: RES2014068CLI).

MDEyeCare app is freely available on iTunes for iPhones and its website (www.mdeyecare.com) describes the method of capturing the image of the eye in a step-wise manner with the help of instruction video. Briefly, the child face is focused on the app screen by touching the face at 3 feet away from the child. Next, the picture is taken when the dial bar shows "perfect" after turning off the light. The app modifies flash and camera settings of smartphone allowing red reflex to be captured in the normal eye and white reflex (Leukocoria) in the affected eye. We initially followed the technique described in the above website. However, we found it necessary to improve the methods as described below. First, an examination room with dimension of about 10 by 10 feet and very dim ambient lighting was created. In order to have pupil dilation, child was made to sit comfortably in the lap of a parent facing the light source and made to wait in this room for about a minute before capturing the image. Examiner sat at a distance of 3 feet facing the child. At least 5 photographs were taken at five gazes; One in straight gaze when child looks at the camera as explained in the app and remaining four gazes when child's attention was taken to lateral and up or down gazes by a colorful ill-defined object or with the help of parents. If this was not possible, then examiner shifted the position of camera in each direction and the images were captured achieving the same result. All the clear images were labeled and stored in the database. Then reflex was analyzed considering color and brightness. No reflex was seen in normal (Fig. 1a) and tumor (Fig. 1c) eye without MDEyeCare app. However, at one end of the extreme is the proper red reflex $(\mathrm{R})$ involving total pupil area in the normal eye (Fig. 1b) and at another end is the proper white-gray reflex (leukocoria) involving whole of pupillary area in RB eye (Fig. 1d). These results were compared with clinical data regarding the number of tumor, tumor size, staging, and location of tumor.

CRADLE app can detect leukocoria during scanning through live view of smartphone camera with illumination from in-built flash. It can also scan through various recreational photographs to detect leukocoria and differentiate from 


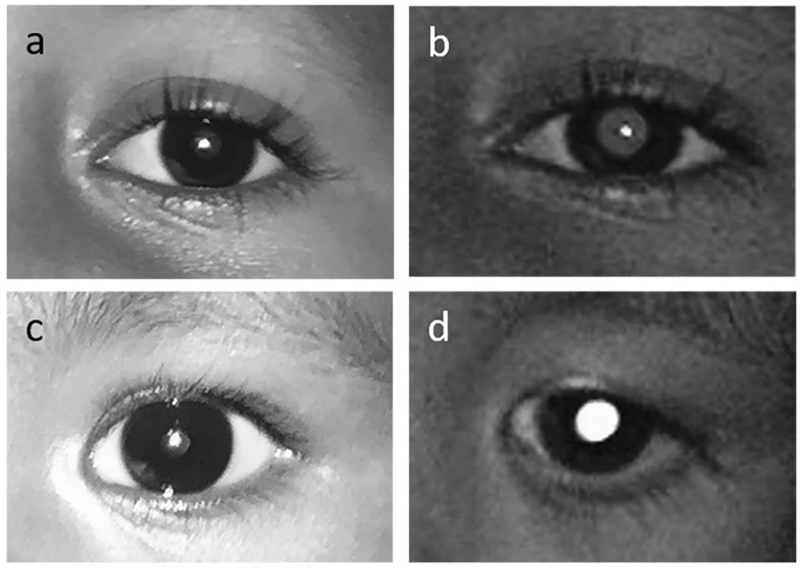

Fig. 1 iPhone 6s images of the left eye of normal (top row) and RB (bottom row) child with and without MDEyeCare app at straight gaze (undilated pupil) photography. Normal eye without app showing (a) no reflex and with app showing red reflex (b). RB eye with group E tumor without app showing no reflex (c) and with app showing leukocoria (d). Normal is 18 months and RB child 7 months old

apparently normal red reflex. The app essentially detects faces and eyes from the photos or video frames, and then analyzes the pupil region for reflex characteristics using machine learning algorithm. After complete analysis, app shows green and red brackets for normal and leukocoric eyes respectively. We screened all of our study population in live mode as well as offline for the photographs taken by iPhone $6 \mathrm{~s}$ with and without MDEyeCare app.

\section{Results}

In a child referred to clinic, white reflex was observed in the image of the right eye taken with iPhone $6 \mathrm{~s}$ without MDEyeCare app at left lateral gaze (Figure 2a2), while no reflex was observed at other gazes as shown in the Figure 2a1, a3-5. However, leukocoria was detected in left (Figure 2b2) and down gazes with MDEyeCare app (Fig. 2b4), which was later confirmed as group $\mathrm{C}$ tumor in infero-nasal quadrant position of the right eye (Fig. 2c, d). Indeed, the direction of gaze that was used to detect leukocoria may suggest the location of tumor at early stage of RB. Further, a clear red reflex was observed in the left normal eye at right gaze (Fig. 2b5) with app in contrast to absence of red reflex (Fig. 2a5) without app.

Another child was referred to the clinic since parents noticed white reflex in one eye. Both eyes were examined using MDEyeCare app. Leukocoria was detected in the right eye in all gazes; while in the left eye, white reflex was observed only in right gaze (Fig. 3). Later, the clinical diagnosis confirmed that the child had bilateral RB (group E right eye and group B left eye). The right eye had tumor dimension of 17 by $16 \mathrm{~mm}$ (MRI) with tumor involving posterior pole. The left eye had two foci of tumors measuring 5-6 mm located beyond vessel arcade in infero and supero nasal quadrant.

Based on the tumor staging as shown in Table 2, eyes were segregated into early (Group A, B, and C) and late (D and E) stages of RB. Among all eyes examined using MDEyeCare app with our method, leukocoria detection rate of $83 \%$ was achieved for group C and $50 \%$ for group B tumor. However, no sign of leukocoria was observed in eyes with group A tumors. In late stage RB, 100\% detection was observed (Table 2). On the other hand, only 4 late stage eyes were picked up by CRADLE app through offline screening of images taken with MDEyeCare app. Among them, only one was detected through live mode and offline image taken with iPhone 6s.

In order to assess the pseudo-leukocoria, which is frequently seen in smartphone images of normal eye caused by flash or the light source of the advanced smartphone camera or reflection off the optic nerve head at off-axis photography [8, 12-14], eyes of four normal children were analyzed with MDEyeCare app. Among images taken at all gazes, two children were showing unilateral leukocoria-like reflex, detected in a photograph at lateral gaze (Supplementary Figure 1). In addition, among the six normal eyes of the unilateral RB patients' images with the app showed pseudo-leukocoria in one patient at lateral gaze photography. Thus, it is possible that reflection of optic nerve head occurs as pseudo-leukocoria only at lateral gaze photography even with MDEyeCare app.

\section{Discussion}

We have demonstrated that iPhone-based MDEyeCare app can be used to detect leukocoria in RB children, without anesthesia and pharmacological dilatation. However, in order to improve the rate of detection in children especially with early stages of RB, we have developed a strategy to achieve $100 \%$ leukocoria detection in children with late stages, $83 \%$ with Group C and 50\% with group B tumor (Table 2). It was possible to detect leukocoria even when tumor diameter as small as $5 \mathrm{~mm}$ and tumors located away from the posterior pole (Fig. 3). Moreover, consistent detection of leukocoria at particular gaze warrants the presence of tumor and also suggests its location. Our approach confirms the earlier study on simulated retinoblastoma eye model [15] which suggested the importance of oblique view red-reflex test to improve the rate of leukocoria detection.

Leukocoria was detected by the family members when the tumor has grown to later stages, thus resulting in enucleation to save the life of patients (Tables 1 and 2). However, early stage tumors were detected in our clinic in bilateral patients and in such cases vision salvage was 

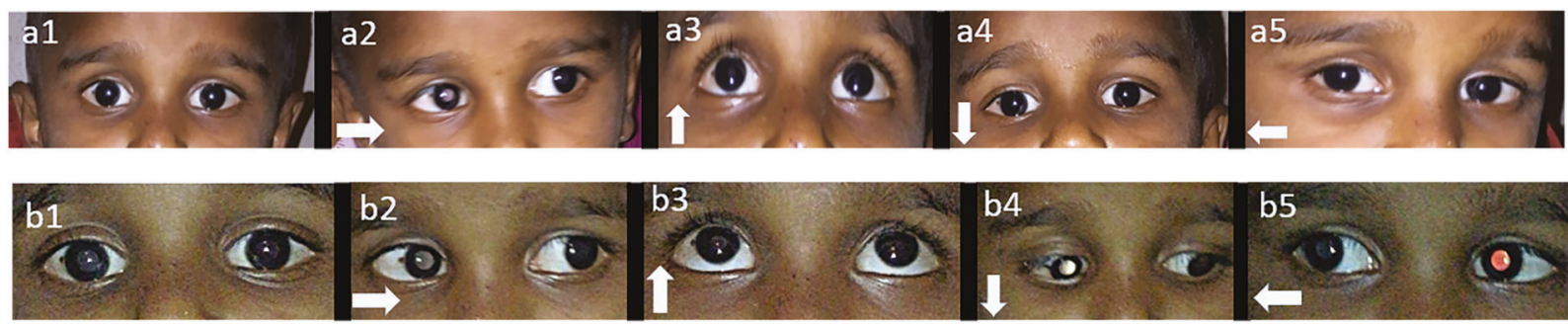

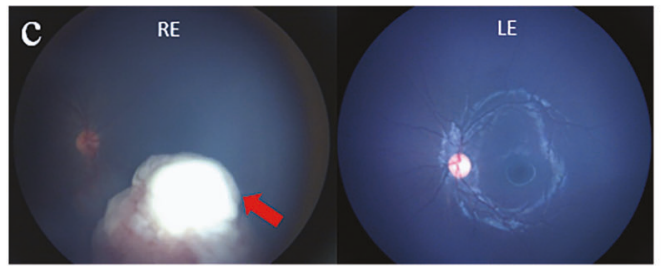

Fig. 2 Images of both eyes of a unilateral patient (33 months) with RB tumor (group C) in the right eye. Photos were taken by iPhone $6 \mathrm{~s}$ camera a without and b with MDEyeCare app in five different gazes (white arrow); showing leukocoria in the right eye in the left gaze (a2). With app, leukocoria was observed in the right eye in both left (b2) and down

possible (unpublished). In this context, the earlier studies $[6,7]$ have clearly demonstrated that detection of RB tumor at the early stages, ensure successful treatment, avoiding the possible enucleation. The parents are unaware of the presence of tumor at the early stage and could detect only after its progression to late stages (Group D and E). Our improved method by using MDEyeCare app, leukocoria can be detected at the early stages of RB, except Group A.

The limitations of the present study in detecting leukocoria are false positives (FPs) and false negatives (FNs). We have observed leukocoria in normal eyes at certain gazes, which might be due to reflection of the optic nerve head at off-axis photography $[8,12,13]$. Though the FP is frequently observed in the smartphone photography [14], the present method with MDEyeCare app showed less frequent unilateral pseudo-leukocoria only at lateral gaze that can be seen as normal red reflex at other gazes (Supplementary Figure 1).

False negatives were observed as red or no reflex type in early stage tumors and no reflex in late stages and thus, it is possible that parents are falsely reassured. Therefore, further improvements in the app and the photographic methods are required. Despite these limitations, our modified method of using MDEyeCare app could help trained paramedical workers (non-ophthalmologists) to suspect a problem in the eye and could refer the children to eye hospital for thorough clinical examination.

In order to achieve the objective of early detection of RB by paramedical workers, we have planned three-pronged strategies: (1) iPhone-based images of the eyes will be taken in Primary Health Centers (PHCs) [16] since babies will be taken to such centers for vaccination several times. (2) The para-medical ophthalmic assistants in Primary Eye Care services (PECs) and Non-governmental organizations/self-help

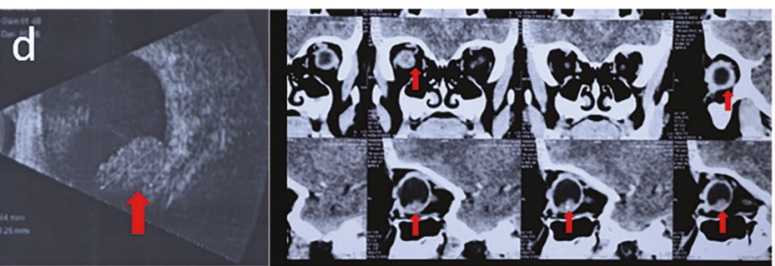

(b4) gazes; red reflex in the left eye (normal) at right gaze (b5). Retcam images of right eye showing group $\mathrm{C}$ tumor in infero-nasal quadrant and left eye with normal fundus (c). USG B-scan of right eye and CT scan of orbit, showing (red arrow) the presence of tumor in the right eye (d), confirming that leukocoria is due to the tumor

groups will be trained for the use of iPhone-based app, the evaluation of images and counseling. Further, Aravind managed Vision Centers will also be involved. Non-ambiguous photographic chart will be provided for referral guidance similar to Muen et al. [17]. (3) To create awareness of the target population, posters showing the images of RB and iPhone-based images will be placed in the above three centers. To start with, this service will be provided in and around Madurai, so that our ophthalmologists could monitor the program.

\section{Conclusion}

Our improved methodology in smartphone-based (iPhone 6s) MDEyeCare App enhanced the detection of Leukocoria (White reflux) due to retinoblastoma even at the early stages. This study carried out in controlled environment showed reliable results confirmed with conventional clinical examination. Therefore, our method will have a significant impact on the visual outcome since early detection is possible without the need for general anesthesia and pharmacological dilation in the setting of Primary Health Centers.

\section{Summary}

\section{What was known before}

- Retinoblastoma (RB) is most commonly referred to the clinic at late stages with white reflex (Leukocoria) in pupil of child as presenting sign, often noticed by family members. 

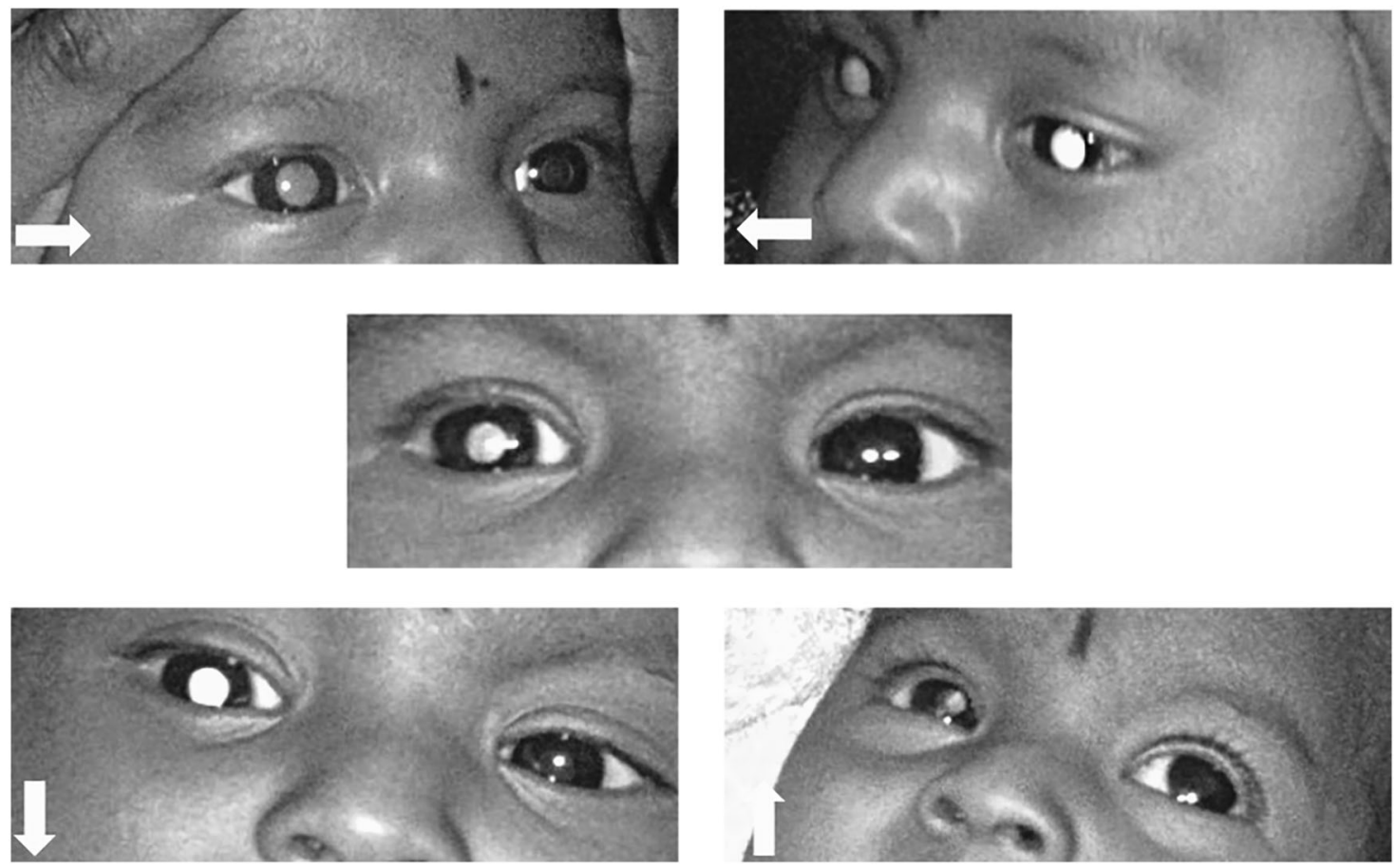

Fig. 3 iPhone 6s Images of bilateral RB patient (3 months) with MDEyeCare app, showing leukocoria in right eye at all gazes, while in left eye at right gaze only. Clinical examination showed group E tumor in the right eye and group B in the left eye

Table 2 Ability of iphone $6 \mathrm{~s}$ MDEyeCare app to detect leukocoria at different stages of RB

\begin{tabular}{lrrrrrr}
\hline Stage & A & B & C & D & E $\begin{array}{l}\text { Total number } \\
\text { of eyes }\end{array}$ \\
\hline Unilateral & 0 & 0 & 2 & 0 & 4 & 6 \\
Bilateral & 4 & 4 & 4 & 5 & 11 & 28 \\
Detected & 0 & 2 & 5 & 5 & 15 & 27 \\
Not detected & 4 & 2 & 1 & 0 & 0 & 7 \\
$\begin{array}{l}\text { Percentage (\%) of } \\
\text { detection }\end{array}$ & 0 & 50 & 83 & 100 & 100 & \\
\hline
\end{tabular}

- Leukocoria can be detected at early stages of RB that would improve better treatment outcome.

- Smartphone-based applications (apps) have been developed to detect leukocoria in the eye of the children with RB tumor through smartphone photography.

\section{What this study adds}

- Validation of smartphone-based leukocoria detection app for the detection of RB at various stages in Indian children without anesthesia and pharmacological dilatation of the pupil has not been done.

- Smartphone-based app (MDEyeCare) with modifications in photography has improved performance in detecting leukocoria of eyes from the RB children and thus can be used by trained paramedical workers for screening of young children and refer to ophthalmic clinic for confirmation.

Acknowledgements We thank Aurolab for financial support (iPhone 6s). We also thank Dr. María Eliana Mánquez for her support and the use of MDEyeCare, and CRADLE app in this study.

\section{Compliance with ethical standards}

Conflict of interest The authors declare that they have no conflict of interest.

Publisher's note: Springer Nature remains neutral with regard to jurisdictional claims in published maps and institutional affiliations.

\section{References}

1. Abramson DH, Dunkel IF, McCormick B. Neoplasms of the eye. In: Holland JF, Bast RL Jr, Morton DL, Frei E III, Kufe DW, Weichselbaum RR, editors. Cancer Medicine. 4th ed. Baltimore, MD: Williams \& Wilkins;1997, p.1517-36

2. Rangamani S, SathishKumar K, Manoharan N, Julka PK, Rath GK, Shanta V, et al. Paediatric retinoblastoma in India: evidence from the National Cancer Registry Programme. Asian Pac J Cancer Prev. 2015;16:4193-8.

3. Broaddus E, Topham A, Singh AD. Survival with retinoblastoma in the USA: 1975-2004. Br J Ophthalmol. 2009;93:24-7. 
4. Seregard S, Lundell G, Svedberg H, Kivelä T. Incidence of retinoblastoma from 1958 to 1998 in Northern Europe: advantages of birth cohort analysis. Ophthalmology. 2004;111: 1228-32.

5. Meel R, Radhakrishnan V, Bakhshi S. Current therapy and recent advances in the management of retinoblastoma. Indian $\mathrm{J}$ Med Paediatr Oncol. 2012;33:80-88.

6. Shields CL, Mashayekhi A, Au AK, Czyz C, Leahey A, Meadows AT, et al. The International Classification of Retinoblastoma predicts chemoreduction success. Ophthalmology. 2006;113:2276-80.

7. Abramson DH, Beaverson K, Sangani P, Vora RA, Lee TC, Hochberg HM, et al. Screening for retinoblastoma: presenting signs as prognosticators of patient and ocular survival. Pediatrics. 2003;112:1248-55.

8. Abdolvahabi A, Taylor BW, Holden RL, Shaw EV, Kentsis A, Rodriguez-Galindo C, et al. Colorimetric and longitudinal analysis of leukocoria in recreational photographs of children with retinoblastoma. PLoS ONE. 2013;8:e76677.

9. Rivas-Perea P, Baker E, Hamerly G, Shaw BF. Detection of leukocoria using a soft fusion of expert classifiers under nonclinical settings. BMC Ophthalmol. 2014;14:1-15.
10. Wolf JA, Moreau JF, Akilov O, et al. Diagnostic inaccuracy of smartphone applications for melanoma detection. JAMA Dermatol. 2013;149:422-6.

11. Linn Murphree A. Intraocular retinoblastoma: the case for a new group classification. Ophthalmol Clin North Am. 2005;18:41-53.

12. Marshall J, Gole GA. Unilateral leukocoria in off axis flash photographs of normal eyes. Am J Ophthalmol. 2003;135:709-11.

13. Russell HC, Agarwal PK, Somner JEA, Bowman RJC, Dutton GN. Off-axis digital flash photography: a common cause of artifact leukocoria in children. J Pediatr Ophthalmol Strabismus. 2011;48:e1-3.

14. Asensio-Sánchez VM, Díaz-Cabanas L, Martín-Prieto A. Photoleukocoria with smartphone photographs. Int Med Case Rep J. 2018;11:117-9.

15. Li J, Coats DK, Fung D, Smith EO, Paysse E. The detection of dimulated retinoblastoma by using red-reflex testing. Pediatrics. 2010;126:e202-7.

16. Rao GN. An infrastructure model for the implementation of VISION 2020: The right to sight. Can J Ophthalmol. 2004;39:589-9.

17. Muen W, Hindocha M, Reddy M. The role of education in the promotion of red reflex assessments. JRSM Short Rep. 2010;1:46. 